\title{
TOTAL SYSTEM ERROR PERFORMANCE OF DRONES FOR AN UNMANNED PBN CONCEPT
}

\author{
R. Geister, L. Limmer, M. Rippl, T. Dautermann, German Aerospace Center, Germany
}

\begin{abstract}
The German Aerospace Center (DLR) operates small drones i.e., octocopters for research purposes at different institutions at different locations. In addition, the DLR is working on the integration of drones into unsegregated airspace in several national and international projects. One of the key elements for a safe integration of drones is the positioning capability of the air vehicle. On the one hand it is required for geofencing applications in order to create no-fly zones and on the other hand it is required to generate an airspace management for unmanned traffic.
\end{abstract}

In recent years, the Performance Based Navigation (PBN) concept was introduced for manned aviation to exploit the navigation performance of modern satellite navigation and to manage the available airspace. One approach for Unmanned Aircraft System (UAS) Traffic Management (UTM) is obviously to transfer the PBN concept for drone applications. However, as drones usually use commercial off the shelf equipment that is usually not certified for aviation applications, the question is how the basic principle can be transferred.

In this work, we used a commercial octocopter (MikroKopter MK Okto XL 6S12 ARF) to assess the horizontal Navigation System Error (NSE) as well as the lateral Total System Error (TSE) while using different GNSS receivers. The horizontal navigation is based on GNSS in stand-alone mode or using SBAS augmentation and a compass for directional information aid. No additional sensors like inertial measurements are used here. We are going to present the results from flight trials with two different GNSS receivers and will draw conclusions for a PBN concept for drones.

\section{Introduction}

Currently, extensive work is being conducted in the area of drone integration into unsegregated airspace. In particular, several activities are developing a traffic management system for unmanned air vehicles. For instance the Unmanned Traffic Management (UTM) and the U-space activities have to be mentioned here. Within the Uspace activities, the project CORUS aims to develop a Concept of Operations (ConOps) for drones operation in the very low level airspace.

One of the major challenges is the definition of the navigation requirements for the operation in the airspace. As there is a large variety of drones, the navigation systems used are also very heterogeneous. Therefore, it might not be feasible to define certain requirements for many different navigation sensors but define requirements for the performance for the navigation systems. This concept is well-defined in manned aviation and it is called Performance Based Navigation (PBN), especially with its implementation called Required Navigation Performance (RNP). The concept will be described in the following chapter.

Nevertheless, one navigation sensor that is common to almost all drones is a Global Navigation Satellite System (GNSS) receiver. In this work, we investigate the navigation performance that can be expected using GNNS within a drone. We used two different GNSS receives for comparison. The observations made can be used to define tangible values for RNP for drones.

Of course, additional constraints have to be considered, for instance degraded GNSS performance in urban environments due to multipath and reduced satellite visibility. Still, our investigations can be used as a baseline for further improvements.

\section{Performance Based Navigation in Manned Aviation}

Giving credit to the multitude of combination of position sources including hybridization of different technologies (like Global navigation 
Satellite Systems (GNSS, [1]), inertial navigation aided GNSS receivers [2], [3], VOR [4], DME [4]), the International Civil Aviation Organization (ICAO) introduced the Performance-based Navigation (PBN) framework. It sets the requirements for a specific airspace or procedure rather than on the equipment used for it. As long as the aircraft can fulfill these requirements it is less important in which way the compliance is achieved.

One current implementation of PBN is called Required Navigation Performance (RNP, [5]) and RNP is a designator for an area navigation system for use within a performance based navigation concept. RNP also includes continuous monitoring of navigation performance and alerting of the pilot in case of failure [6]. Only lateral (or cross-track) RNP accuracy is indicated by a number following the letters RNP (i.e., RNP 0.3 for $0.3 \mathrm{NM}$ accuracy). In this case, accuracy relates to the Total System Error (TSE) which is a combination of the Flight Technical Error (FTE) and the Navigation Sensor Error (NSE), and designates the 95\% uncertainty bounds for the accuracy of the navigation solution. An integrity bound of 2xRNP is used to ensure containment of the flight path for contingency reasons.

Usually, the pilot is provided with a course deviation indicator (CDI) which shows the rectilinear cross track deviations from the desired track. For the vertical component the aircraft uses a barometric altimeter to determine its height above a designated pressure surface. The vertical TSE is not covered under RNP, but altimeter NSE accuracy requirements are specified.

With the advent of RNP, an additional leg type was created to enable precise path following during such curved segments, called the radius-to-fix (RF) path terminator. Fig. 2 from [7] schematically illustrates a RF leg. The path begins at a fix, follows a circle with a predefined radius and center and ends at a second fix. The exit track angle of the RF must be the same as the one of the following leg in order to avoid discontinuities. Following a curved flight path precisely requires the autopilot to have roll steering capability and a direct connection to the FMS. If manually flown, the pilot must either be provided with a bank angle target or the omnibearing selector on the horizontal situation indicator must continuously be adjusted into the direction of turn at velocity of the rate of turn [8].

In order to use RF during final approach and in the initial and intermediate phase of the missed approach, additional training of the crew and higher requirements for aircraft equipment are imposed by all regulators and the approaches are published as RNP AR APCH (here, AR stands for Authorization Required). The FAA implementation RNP AR is called RNP Special Aircraft and Aircrew Authorization Required (SAAAR) [9] and the history of its implementation can be found, for example in [10].

RNP can be used for all phases of flight, including instrument approaches. When the navigation solution is augmented by a Satellite Based Augmentation System (SBAS), precision final approach guidance can be achieved. This is called Localizer Performance with Vertical guidance (LPV).

\section{PNB Concept for Drones}

The main question remaining is if all aspects of the PBN concept can be transferred to drone operation, specifically small drones operating in the very low level (VLL) environment. Is it feasible to assign certain RNP values to certain airspaces for drone operations? Currently, the drone community differentiates between rural areas, where the risk of injury or damage of ground impact of drones is rather small and urban areas where this risk is rather high. In contrast, GNSS availability might be increased in rural areas due to the absence of buildings that can influence signal reception in the drones. On the other hand, it is expected that drone traffic will be higher in urban areas; hence an increased navigation performance is required.

To be able to answer the question above, the two major error components have to be analyzed. On the one hand the NSE, which is in our case given by the GNSS performance (as we are only investigating GNSS performance without sensor fusion) and on the other hand the FTE which is given by the drone pilot, the automatic flight system and the type of the drone. It is assumed that more agile drones like multicopters have a smaller FTE than fixed wing drones. 


\section{Expected NSE performance of Receivers}

The GNSS receivers used on current drones on the market originate in mass market and are designed to perform robustly under very adverse reception conditions, while consuming as little power as possible. The specifications for such types of receivers are dictated by the market or set by the manufacturer.

For example, modern GNSS chips include methods on signal level that include estimation of multipath components in the received signal, vector tracking technologies and other filtering extensions that help fight against poor reception around and in buildings.

In contrast, aviation receivers are designed with a single reception environment in mind: An external antenna on top of an aircraft. Signal obstruction by nearby buildings is not an issue, and multipath reflections originate only from the aircraft itself or from ground. It has to be noted that the area in which an aircraft is allowed to use GNSS measurements as an input to instrument operations such as a precision approach has to fulfill requirements with respect to signal propagation, similar to the Cat-I/Cat-III zones around runways that exist to avoid ILS signal reflections.

The immediate environment of drones would have to be considered significantly different from that of a civil aircraft. Proximity to ground and surrounding buildings or other structures add many potential reflections that create multipath errors in the measured ranges. On top, the RF environment for this use case may have to be considered less protected than for the typical aviation user today.

The above differentiators do not preclude the provision of a navigation error model that suits the needs of a PBN-like concept. From an integrity perspective, two major adaptations seem necessary:

- The use of commercial off-the-shelf, consumer grade GNSS hardware is better designed for use on a drone on a signal processing level. However other design requirements such as low power consumption may trade in reliable positioning capability and thus prevent integration into an integrity-oriented navigation system.
- Multipath and RF interference may be very different from the typical environment that the original PBN based GNSS concept was defined in. Modeling of multipath error envelopes may not be sufficient to achieve good performance; instead, large multipath errors may have to be detected and excluded by the aid of additional sensors in a filter. This approach however increases complexity and may obstruct the path to certification of the navigation system.

\section{Expected FTE performance of drone systems}

Looking at the FTE, it is driven either by the drone pilot together with the flight control system as well as the indication of the actual and the desired flight track. Or it is driven by the auto-flight system in conjunction with the flight control system. As the manual FTE is strongly dependent on the drone pilot and the equipment of the drone (first person view capabilities, flight path indication), it is not considered in this work.

Therefore, only automatic flight systems are considered. In addition, the type of the drone has to be taken into account. For instance, multicopters are considered to be more agile than fixed wing drones. They are able to execute sharper turns while staying close to the desired flight path.

There are several autopilot systems for drones on the market. They can be closed box systems developed by the drone manufacturer but there also a few open source systems available. As those systems are also very diverse, it is hard to declare an expected value for the FTE in general. Therefore, we assume that even if drones will not have to be certified like manned aircraft, there will have to be a certain certification for the automatic flight system if a drone intends to use drone-RNP airspace. In this work, we investigated the FTE of a certain drone that might be representable for the class of mulitcopters but more trials with different drones will have to confirm this.

\section{Test Setup}

For the conducted flight tests, we used an octocopter, called MikroKopter MK Okto XL 6s12 ARF [11], to implement and validate the navigation performance generated by different GNSS 
receivers. The drone is powered by eight electrical engines with a thrust of $2400 \mathrm{~g}$ per unit which allows carrying payload up to $4 \mathrm{~kg}$. The carbon fiber chassis is dimensioned with $735 \times 735 \times 450 \mathrm{~mm}$.

Equipped with two lithium polymer accumulators with a capacity of $4500 \mathrm{mAh}$ flight durations up to twenty minutes are possible depending on flight path characteristics and carried payload.

The flight control is provided with technical aids to enable flight manually by the remote pilot as far as fully automatically flown maneuvers and flight paths. To support the pilot during manual operations the octocopter is prepared with functions like position hold and care free (fixation of orientation independent of yaw angle).

Prevention of crashes in cases of e.g. used energy or loss of connection to the ground station safety procedures like failsafe (automatic approach of defined position or home position) or coming home are implemented.

Performing automatic operations is ensured by waypoint flight. Up to 100 predefined coordinates can be flown while monitoring by remote pilot.

Sensor based data for navigation and flight position measurement are supplied by acceleration sensors, gyroscopes, magnetic compass, barometric altimeter and a GPS receiver with antenna.

The basic system of the octocopter uses the MK GNSS V4 module for GNSS navigation which is a combination of GNSS antenna, GNSS receiver and magnetic compass. Receiving GNSS signals is provided by the U-Blox NEO-M8Q [12] receiver that allows using GPS, QZSS, BeiDou and GLONASS (just GPS satellites were used during the flight tests) as well as SBAS.

In order to have reference data for validation of the navigation performance after the flight tests, it was required to equip the drone with additional measurement sensors. Therefore, the Javad Sigma QM [13] was installed enabling position measurement with high precision. Ancillary the American and European GPS and Galileo the functional range ensures receiving GLONASS, QZSS and BeiDou satellites. Furthermore, the Sigma QM is suitable for SBAS (Satellite Based Augmentation Services) and carrier phase measurement. The reference system is completed by an additional GNSS antenna, called Maxtena M1227HCT-A2-SMA [14], applicable for high precision solutions. It is an active system with helix architecture constructed to receive L1 and L2 carrier frequencies.

Developing the test flight scenarios was done by using the specially provided software MikroKopter-Tool [15]. The tool enables the user to program routes up to 100 waypoints with different options during approaching or holding waypoints like observation of a defined target as well as performing varying kinds of velocities or climbs and descents. All in all, seven flight paths were created with MikroKopter-Tool to investigate the navigation performance in realistic operation scenarios such as sector search pattern or creeping line pattern [16] for observation and investigation missions. One example is given in Figure 1.

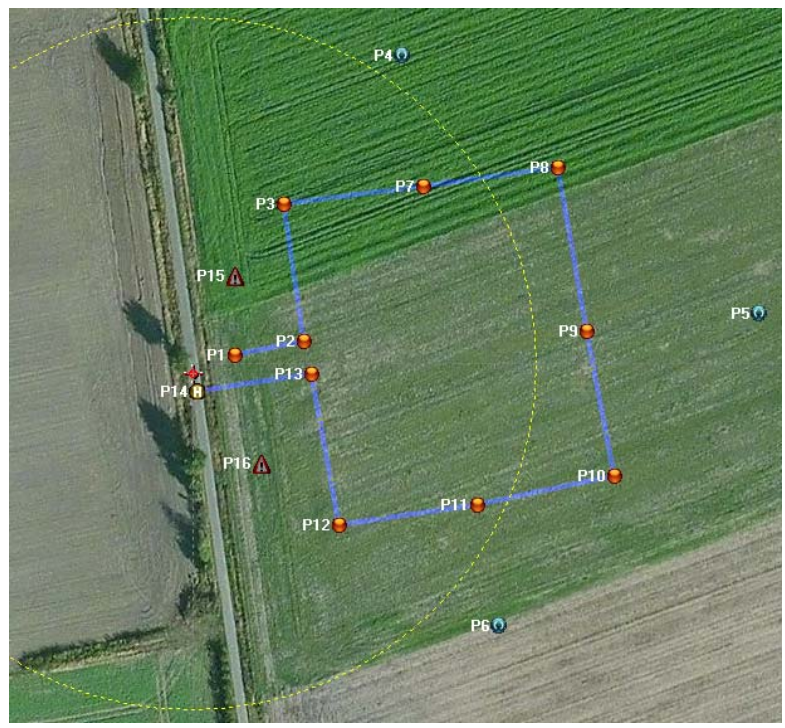

Figure 1. Example of designed flight path (flight path a)

Navigation performance is determined in two scenarios performed by using two different GNSS receiver. In the first step the previous mentioned receiver of the base system will be analyzed with Sigma QM post processed data as reference position system. Second scenario will be transacted with Sigma QM supplying non-post-processed data during flight for the flight control and recorded values for post processing as reference measurement. 
For the analysis of the results, we used the software GrafNav to retrieve the drone's "true" position in post-processing [17].

For the calculation of the FTE and TSE own developed software as well as the PEGASUS toolset [18] was used.

\section{Test Results}

Two different flight trials were conducted. For the first one, the on-board receiver was used to navigate the drone. For the second one, the Javad receiver was used [19].

In addition, two different error components were investigated. On the one hand, the horizontal $\mathrm{NSE}_{\mathrm{Hor}}$ was investigated. This error is given by the distance between the estimated position of the navigation system during the flight and the true position of the post processing solution at that given point of time. On the other hand, the flight path following accuracy was investigated. This was done by using the cross track NSE $_{\mathrm{CT}}$ and the lateral FTE. The lateral deviation of the drone's Actual Flight Path (AFP) from the Desired Flight Path (DFP) is the Total System Error (TSE). Figure 2 shows the TSE with its two main components.

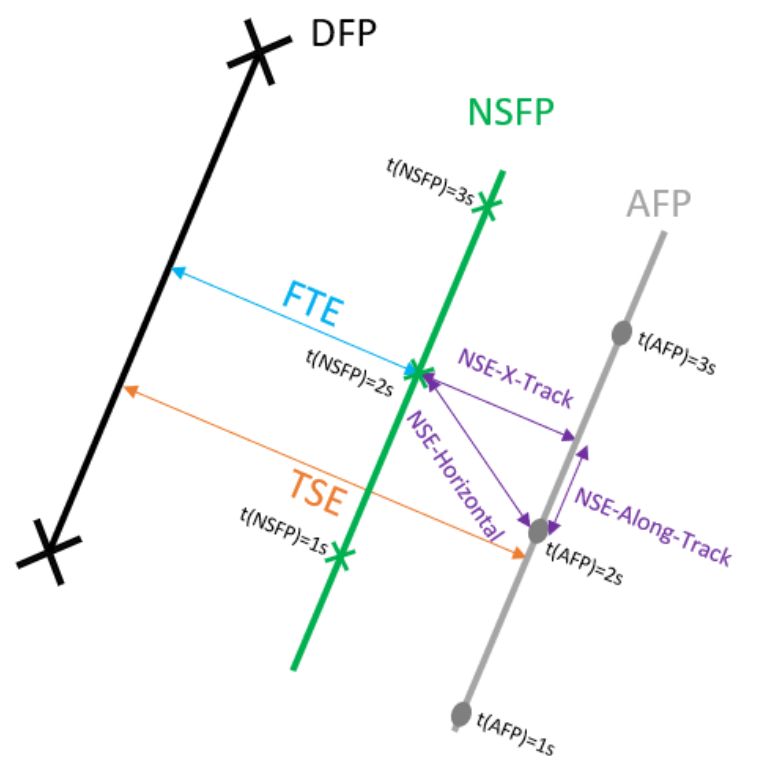

Figure 2. Definition of the TSE

As the data recording did not have the same rate, some of the points had to be interpolated, which can introduce an additional (small) error. In addition, the weather conditions were different for the two conducted trials. During the second trial, the ground wind velocity was approx. 5kts higher than during the first trial (13kts compared to 8kts). As we used heights up to $90 \mathrm{~m}$, the difference in wind velocity is supposed to be even higher.

Table 1 shows the results for flight trial 1 . The arithmetic mean error value as well as the maximum and the minimum value of the horizontal $\mathrm{NSE}_{\mathrm{Hor}}$ observed are given for a given flight path. It can be seen that the mean error is roughly between one meter and $7.5 \mathrm{~m}$. It is also visible, that the variance of the errors is rather high. In addition, the error is strongly dependent on the velocity of the drone during the flight.

Especially during flight path $1 \mathrm{c}, 1 \mathrm{~d} 1$ and $1 \mathrm{~d} 2$ the drone was travelling with maximum velocity. This directly leads to an increased $\mathrm{NSE}_{\mathrm{Hor}}$. In theory, the NSE $\mathrm{H}_{\mathrm{Hor}}$ should not be dependent on the velocity but it seems that either the on board receiver and the reference receiver use some internal extrapolation in order to stabilize their position or there is as issue with the accuracy of the timestamps. In addition, it was observed, that the on board receiver is always lagging compared to the post processed reference position. The observed error is therefore mainly an along track error.

Table 1. Observed NSE $\mathrm{Hor}_{\text {during trial } 1}$

\begin{tabular}{|l|c|c|c|}
\hline Horizontal NSE in m & Mean & Max & Min \\
\hline Flight path 1a & 1.68 & 7.92 & 0.28 \\
\hline Flight path 1b & 2.39 & 12.29 & 0.01 \\
\hline Flight path 1c & 7.41 & 19.72 & 0.12 \\
\hline Flight path 1d1 & 3.45 & 19.46 & 0.04 \\
\hline Flight path 1d2 & 6.50 & 20.43 & 0.21 \\
\hline Flight path 1e1 & 1.48 & 3.39 & 0.58 \\
\hline Flight path 1e2 & 0.92 & 6.77 & 0.06 \\
\hline
\end{tabular}

This behaviour can be observed in Figure 3 . The errorbars represent the magnitude of the horizontal $\mathrm{NSE}_{\mathrm{Hor}}$. During the long legs, the drone is flying at maximum velocity and the NSE increases. During the short legs, the drone is flying

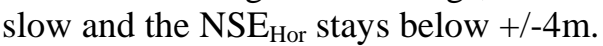




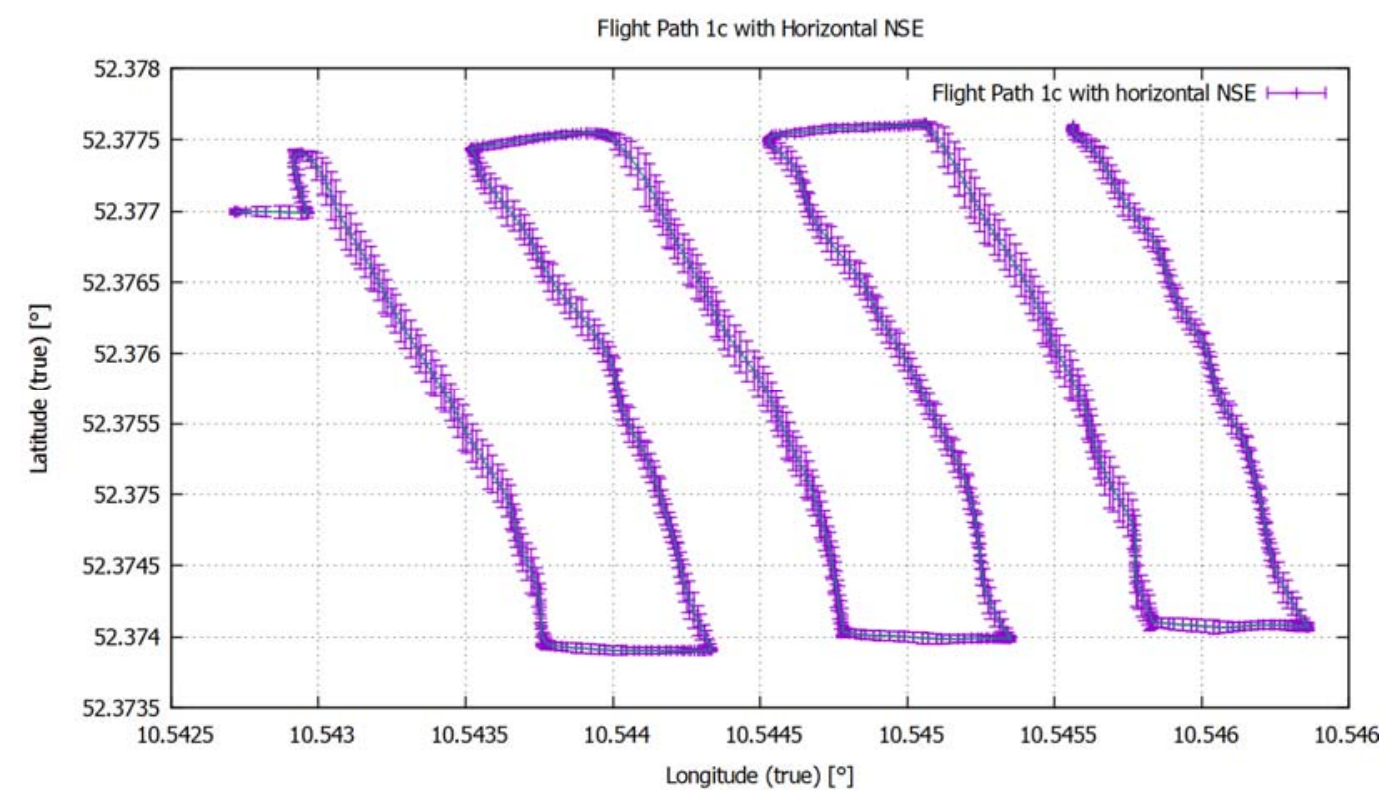

Figure 3. $\mathrm{NSE}_{\mathrm{Hor}}$ during flight path $1 \mathrm{c}$ in $\mathrm{m}$

Table 2 shows the results for flight trial 2. It can be seen, that the mean $\mathrm{NSE}_{\mathrm{Hor}}$ was generally smaller and also the maximum error was smaller than in trial 1. The Javad receiver with the additional antenna was able to reduce the $\mathrm{NSE}_{\mathrm{Hor}}$ and especially the variance of the $\mathrm{NSE}_{\mathrm{Hor}}$ significantly in some cases. However, it has to be noted that this comes with the price of increased weight for the receiver and the antenna.

Table 2. Observed NSE $_{\mathrm{Hor}}$ during trial 2

\begin{tabular}{|c|c|c|c|}
\hline $\begin{array}{l}\text { Horizontal } \text { NSE }_{\mathrm{Hor}} \text { in } \\
\text { m }\end{array}$ & Mean & Max & Min \\
\hline Flight path 2a & 1.02 & 6.02 & 0.04 \\
\hline Flight path $2 \mathrm{~b}$ & 1.16 & 6.11 & 0.08 \\
\hline Flight path 2c & 3.07 & 8.84 & 0.16 \\
\hline Flight path 2d1 & 1.43 & 6.95 & 0.03 \\
\hline Flight path $2 \mathrm{~d} 2$ & 2.33 & 8.97 & 0.04 \\
\hline Flight path 2e1 & 1.09 & 2.22 & 0.07 \\
\hline Flight path 2e2 & 0.70 & 3.38 & 0.04 \\
\hline
\end{tabular}

Next to the horizontal $\mathrm{NSE}_{\mathrm{Hor}}$, the flight path following accuracy was investigated during the two flight trials. Here, as described above, the lateral (cross-track) $\mathrm{NSE}_{\mathrm{CT}}$ and the FTE are analyzed.
Together, they form the TSE. Table 3 shows the Root Mean Sqare (RMS) error observed for the $\mathrm{NSE}_{\mathrm{CT}}$, FTE and TSE during flight trial 1.

Table 3. Observed TSE during trial 1

\begin{tabular}{|l|c|c|c|}
\hline $\begin{array}{l}\text { RMS error of error } \\
\text { components in m }\end{array}$ & NSE $_{\text {CT }}$ & FTE & TSE \\
\hline Flight path 1a & 0.76 & 0.74 & 1.51 \\
\hline Flight path 1b & 0.57 & 2.52 & 2.24 \\
\hline Flight path 1c & 1.30 & 5.75 & 5.33 \\
\hline Flight path 1d1 & 1.27 & 4.05 & 3.82 \\
\hline Flight path 1d2 & 1.05 & 6.11 & 6.05 \\
\hline Flight path 1e1 & 0.76 & 1.69 & 1.06 \\
\hline Flight path 1e2 & 0.65 & 2.25 & 2.62 \\
\hline
\end{tabular}

It can be seen that the $\mathrm{NSE}_{\mathrm{CT}}$ is smaller now as only the cross-track component is taken into account. It I also visible, that generally the FTE is larger than the $\mathrm{NSE}_{\mathrm{CT}}$ and also increases when the drone is travelling faster (e.g., during flight paths 1c, $1 \mathrm{~d} 1$ and $1 \mathrm{~d} 2$ ). The RMS values for the TSE are generally slightly smaller than the FTE because the NSE $\mathrm{CT}_{\mathrm{CT}}$ and FTE can have cancelling effect if they are to the 
different sides of the desired flight path. The maximum value for the TSE is around $6 \mathrm{~m}$ here.

Table 4 thows the same data for flight trial 2 . Again, the navigation system was generally able to reduce the NSE but not as much as in the earlier case as only the lateral component is taken into account. In contrast to that, the FTE and the resulting TSE was generally increased in flight trial 2 . In some cases the FTE and the TSE values are twice as big as during flight trail 1. Possible explainations are given in the next section.

Table 4. Observed TSE during trial 2

\begin{tabular}{|l|c|c|c|}
\hline $\begin{array}{l}\text { RMS error of error } \\
\text { components in m }\end{array}$ & NSE $_{\text {CT }}$ & FTE & TSE \\
\hline Flight path 2a & 0.64 & 4.42 & 3.90 \\
\hline Flight path 2b & 0.59 & 6.17 & 5.64 \\
\hline Flight path 2c & 0.69 & 9.85 & 9.40 \\
\hline Flight path 2d1 & 0.62 & 5.97 & 5.51 \\
\hline Flight path 2d2 & 0.67 & 5.37 & 5.01 \\
\hline Flight path 2e1 & 0.84 & 4.46 & 3.78 \\
\hline Flight path 2e2 & 0.50 & 4.29 & 3.99 \\
\hline
\end{tabular}

\section{Discussion}

In order to discuss the results, the direct comparison of the observed values is given in Table 5. The table shows that the horizontal $\mathrm{NSE}_{\mathrm{Hor}}$ can be reduced when using a high end, dual frequency GNSS receiver. However, the receiver used, comes with the additional burden of weight and a dual frequency antenna. It has to be stated however, that this receiver is not optimized for the use in a drone. Therefore, the weight can be significantly reduced.

Table 5. Horizontal NSE ${ }_{\mathrm{Hor}}$ comparison

\begin{tabular}{|c|c|c|c|}
\hline $\begin{array}{ll}\text { Horizontal } & \text { NSE }_{\text {Hor }} \\
\text { in } \mathbf{~ m} & \end{array}$ & $\begin{array}{c}\text { Mean } \\
\text { flight } \\
\text { trial } \\
1\end{array}$ & $\begin{array}{c}\text { Mean } \\
\text { flight } \\
\text { trial } \\
2\end{array}$ & Diff. \\
\hline Flight path a & 7.92 & 6.02 & 1.90 \\
\hline Flight path b & 12.29 & 6.11 & 6.18 \\
\hline Flight path c & 19.72 & 8.84 & 10.88 \\
\hline Flight path d1 & 19.46 & 6.95 & 12.51 \\
\hline Flight path d2 & 20.43 & 8.97 & 11.45 \\
\hline Flight path e1 & 3.39 & 2.22 & 1.16 \\
\hline Flight path e2 & 6.77 & 3.38 & 3.40 \\
\hline
\end{tabular}

As stated before, the main error component of the horizontal NSE is the along track error. This effect is visible in both flight trials. One possible explanation is an internal extrapolation of the position of the receivers in order to stabilize the output. Interestingly, the on-board receiver lags behind the reference position whereas the Javad position is always ahead of the reference position. Another possible reason is an issue with the accuracy of the timestamps either of the on-board receiver or the reference receiver. The reason for this behavior still has to be investigated.

Table 6 shows the RMS values of the TSE during flight trial 1 and flight trail 2 as well as their difference. As described before, the TSE was generally bigger during flight trail 2 due to the increase of the FTE allthough the $\mathrm{NSE}_{\mathrm{CT}}$ was generally smaller. The reason for this behavior could have different reasons. On the one hand, the wind conditions were different during the trials. The wind was stronger during the second trial. In addition, during the second trial, one propeller had to be replaced on the drone. This replacement was not made from carbon fibre than the other seven and in additioon had a smaller diameter. This might have a slight impact on the flight control system as it generates a different ammount of lift. Allthogh the drone is able to fly that way, or even with one motor not operating, this could lead to an increased FTE. Another possible explaination could be the integration of the Javad receiver. In order to use the navigation solution of the Javad, the on-board interface software had to be changed in order to accept the new format.

Table 6. TSE comparison

\begin{tabular}{|l|c|c|c|}
\hline $\begin{array}{l}\text { Lateral TSE in m } \\
\text { (RMS) }\end{array}$ & $\begin{array}{c}\text { Mean } \\
\text { flight } \\
\text { trial } \\
\text { 1 }\end{array}$ & $\begin{array}{c}\text { Mean } \\
\text { flight } \\
\text { trial } \\
\mathbf{2}\end{array}$ & Diff. \\
\hline Flight path a & 1.51 & 3.90 & -2.39 \\
\hline Flight path b & 2.24 & 5.64 & -3.40 \\
\hline Flight path c & 5.22 & 9.40 & -4.18 \\
\hline Flight path d1 & 3.82 & 5.51 & -1.69 \\
\hline Flight path d2 & 6.05 & 5.01 & 1.04 \\
\hline Flight path e1 & 1.06 & 3.78 & -2.72 \\
\hline Flight path e2 & 2.62 & 3.99 & -1.37 \\
\hline
\end{tabular}


This change led to some minor timing issues. The update rate of the navigation was slightly higher than during flight trial 1 . This could have an impact on the processing load of the drone's flight control system.

In order to investigate the reasons and in order to further investigate the wind effects on the FTE, additional flight trials are envisaged. However, the results and the issues show that there could be a great variance in TSE performance of drones even if only different meteorological conditions are considered. The use of additional sensors and sensor fusion could lead to even smaller NSE $\mathrm{E}_{\mathrm{CT}}$ but it is doubtful that this would reduce the FTE.

Based on the results, it can be stated that in our case, just like in manned aviation, generally the FTE is the determining factor for the TSE. This would then be the main factor for the drone-RNP airspace. Therefore, the flight control system needs to be optimized for flight path following in order to comply with the RNP requirements.

During our trials we observed lateral TSE values (RMS) between $1.06 \mathrm{~m}$ and $9.40 \mathrm{~m}$. Considering the worst case scenario, we were able to show an RNP compliance of approx. $10 \mathrm{~m}$. This could be a reference for future drone airspace design.

In addition to the navigation accuracy, the monitoring and alerting has also to be considered. How can the integrity be ensured? Through the use of SBAS, protection levels are available for an upper bound of the NSE but only for aviation users in controlled environment. The integrity concept used in SBAS could however be extended to support also users in high-multipath environment, under less stringent integrity requirements. However the availability of SBAS in urban environments will be limited if a Geostationary Satellite Link is used. Therefore, contingency plans have to be considered during the airspace design such as provision of the SBAS signal through terrestrial digital communication channels, e.g. LTE/5G. The upper bound of the FTE could also be an issue because it is very likely that automatic flight systems would need to be validated and certified in order to ensure FTE performance. This could lead to additional costs for drone manufacturers or drone operators if a certain performance is required.

\section{Conclusion}

In this work, we showed the lateral TSE behavior of a multicopter with two different GNSS receivers. In two flight trials we showed the differences in the error components. In conclusion, specific values for the navigation and flight path following performance of this particular drone can be deduced. A maximum TSE of approx. $10 \mathrm{~m}$ was observed depending on the desired flight path and the wind conditions. Following the RNP concept for manned aviation, an integrity bound of 2xRNP would lead to a drone-RNP integrity bound value of approx. $20 \mathrm{~m}$ here. The nominal RNP value would be $10 \mathrm{~m}$ in our case. The TSE would have to be smaller than this value in $95 \%$ of the flight time.

For the construction of airspace, in particular drone-RNP airspace, this value could be used. A value of $20 \mathrm{~m}$ could already be too high for urban applications and signal degradation is not taken into account here. In addition, more wind conditions would have to be investigated to confirm the observed TSE value.

Due to the vast diversity of drones on the market, we assume that the drone-RNP airspace would be designed independently of a certain type of drone but due to the limitations in an, for instance, urban environment. The resulting value would be the TSE value that a drone must be able to comply with in $95 \%$ of the flight time. If a drone is not able to do so, it wouldn't be able to use the airspace. Additional parameters like vertical separation and detect and avoid capabilities can also be factored in to increase the accessibility of airspace.

This approach would likely lead to some form of certification process for at least the autopilot system. This could lead to an increase of cost for drone manufactures or operators. This has to be taken into account when investigating use cases for drones especially in an urban area.

\section{References}

[1] P. Misra and P. Enge, Global Positioning System: Signals, Measurements, and Performance. No. ISBN 0-9709544-1-7, Ganga-Jamuna Press, 2011.

[2] D. H. Titterton and J. L. Weston, Strapdown Inertial Navigation Technology, 2nd Edition. The Institution of Electrical Engineers, 2004. 
[3] P. Groves, Principles of GNSS, Inertial, and multisensor integrated Navigation Systems. Artech House Inc, 2007.

[4] B. Forssell, Radionavigation Systems. GNSS Technology and Applications Series, Artech House, 2008.

[5] R. J. Kelly and J. M. Davis, "Required Navigation Performance (RNP) for precision approach and landing with GNSS application,” Navigation, vol. 41, no. 1, pp. 1-30, 1994.

[6] ICAO, Doc 9613, Performance-Based Navigation Manual, Fourth Edition, 2013.

[7] R. Geister, T. Dautermann, V. Mollwitz, C. Hanses, H. Becker, 3D-Precision curved approaches: a cockpit view on ATM, in: Proceedings of the Tenth USA/Europe Air Traffic Management Research and Development Seminar (ATM2013), 2013.

[8] Garmin, Garmin radius to fix leg project report, Tech. Rep. Garmin Document 005-00586-21, Rev.1, Garmin International, Inc, Jan 2013.

[9] United States Standard for Performance Based Navigation (PBN) instrument procedure design, FAA Order 8260.58, Sept. 2012.

[10] S. Miller, J. Bruce, Integration of the constant radius $A R C$ to a fix (RF) navigation leg type into NextGen, in: 2011 IEEE/AIAA 30th Digital Avionics Systems Conference (DASC), 2011, pp. 4B1-1-4B110.
[11] https://www.mikrocontroller.com/index.php? main_page=product_info\&products_id=840, accessed march 2018

[12] HiSystems GmbH, operating manual MK OktoXL 6S12, V01 issue 06/17, 2017

[13] Javad GNSS, „SigmaQM Data Sheet, http://www.javad.com/downloads/javadgnss/sheets/S igmaQM_Datasheet.pdf, accessed march 2018

[14] http://de.farnell.com/maxtena/m1227hct-a2sma/antenne-1-217-1-25-1-565-1-61ghz/dp/2484959, accessed march 2018

[15] http://wiki.mikrokopter.de/MikroKopterTool, accessed march 2018

[16] D. Geister, G. Schwoch und H. Becker, „Flight Testing of Optimal Remotely-Piloted-AircraftSystem Scan Patterns, “ Journal of Aircraft 54 (5), pp. 1675-1691, 2017.

[17] www.novatel.com/products/software/grafnav, accessed march 2018

[18] http://www.eurocontrol.int/pegasus, accessed march 2018

[19] L. Limmer, Modification and preparation of a multicopter for precise landings and validation of its navigation accuracy through flight trials, thesis, 2017

\section{Integrated Communications Navigation and Surveillance (ICNS) Conference} April 10-12, 2018 